\title{
Stochastic resonance in a Hodgkin-Huxley neuron in the absence of external noise
}

\author{
D. T. W. Chik, ${ }^{1}$ Yuqing Wang, ${ }^{1,2}$ and Z. D. Wang ${ }^{1,3, *}$ \\ ${ }^{1}$ Department of Physics, University of Hong Kong, Pokfulam Road, Hong Kong, People's Republic of China \\ ${ }^{2}$ Department of Mathematics, University of British Columbia, Vancouver, Canada \\ ${ }^{3}$ Department of Material Science and Engineering, University of Science and Technology of China, Hefei, China
}

(Received 11 December 2000; revised manuscript received 18 April 2001; published 26 July 2001)

\begin{abstract}
We study numerically nonlinear responses of a periodically forced Hodgkin-Huxley neuron. The coherence of the system in the absence of external noise, namely, the "intrinsic stochastic resonance," is evidenced by the multimodal aperiodic firing pattern, a bell-shaped curve in the signal-to-noise ratio, and the statistical features of the mean firing rate. The subthreshold intrinsic oscillations enhance the signal transduction in a manner different from that in models studied previously.
\end{abstract}

DOI: 10.1103/PhysRevE.64.021913

PACS number(s): 87.19.La, 87.10.+e, 05.45.Pq

One major motivation to study stochastic resonance (SR) is its application in biology, and particularly in neuroscience. $\mathrm{SR}$ is a process that consists of a noise-induced enhancement of the response of a nonlinear system to a weak, external, time-periodic modulation in the presence of background noise. The signal-to-noise ratio (SNR) is maximized by a nonzero value of the applied noise. Neurons are inherently stochastic devices for information processing and are efficient in detecting a weak signal. It has been shown both experimentally and theoretically that noise can enhance weak signal transduction in sensory neurons via SR [1]. In addition to direct evidence, when a bell-shaped SNR curve is clearly seen by tuning external noise, another kind of experimental indication for SR is that, in a system of periodically forced sensory neurons in situ where the external noise cannot be tuned or even identified [2], a multimodal interspike interval histogram (ISIH) (in which the time intervals between successive spikes are assembled into a histogram) exhibits a remarkable resemblance to the residence-time distribution of bistable systems driven by a weak periodic signal in the presence of external noise [3]. Recently, it was found that, in the absence of noise, such a multimodal ISIH can still be obtained if the excitability of the neuron is taken into account [4-6]. It is the subthreshold intrinsic oscillation, instead of external noise, that enhances signal transduction, and the irregularity residing in the spike train can be attributed to deterministic chaos. Also, a nonzero SNR has been reported in several experiments when the external noise is switched off [7-9], which is assumed to stem from the existence of intrinsic or internal noise. Such nonlinear features of excitable oscillators may be viewed as a kind of "intrinsic stochastic resonance" (ISR), namely, stochastic resonance in the absence of external noise. The ISR has been demonstrated by a bell-shaped SNR curve in the absence of external noise if the bistable discrete map falls into the chaotic region [10]. The multimodal ISIH in the absence of external noise was addressed in Ref. [4] by using the FitzHugh-Nagumo (FHN) model. Recently, ISR near a period doubling bifurcation was analyzed in the Hindermarsh-Rose (HR) model, by using the

\footnotetext{
*Author to whom correspondence should be addressed. Email address: zwang@hkucc.hku.hk
}

SNR, maximum Lyapunov exponents, the ISIH, and the mean firing rate $[5,6]$. Indeed, ISR was observed in an experiment on a giant squid axon [4].

Biologically, the excitability of neural systems plays a fundamental role in neural information processing. The role of external noise in excitable neural models has been discussed in recent years $[9,11-13]$. On the other hand, the excitability of neurons can lead to intrinsic sub- and suprathreshold oscillations as well as deterministic chaos, which appear to have intrinsic stochasticity in neural systems [14]. Unlike external noise, such intrinsic stochastic behavior can be tuned by the neural system itself through physiochemical changes, e.g., through the effect of neuromodulators. Recently, it was claimed that neuromodulation is important in understanding signal transduction, pattern formation, and even higher biological functions, such as metalearning and emotion [15]. In this paper, we study ISR using the HodgkinHuxley $(\mathrm{HH})$ neural model, which serves as a paradigm for modeling spiking neurons [16]. We show how a weak signal is enhanced by tuning the subthreshold intrinsic oscillation. It is also indicated that the ISR in the present HH model is different from that in high-order nonlinear oscillator models, as their patterns of intrinsic oscillation are different. More importantly, since the $\mathrm{HH}$ model is biologically realistic, we may attribute the ISR to the inherent membrane properties of neurons, enabling us to understand relevant experiments more reasonably.

The HH model, which is based on the nonlinear conductance of ion channels, is described by a set of four timedependent variables $(V, m, n, h)[16]$,

$$
\begin{gathered}
\frac{d V}{d t}=f(V, m, n, h)+I_{0}+I_{1} \sin (2 \pi f t), \\
\frac{d m}{d t}=\frac{m_{\infty}(V)-m}{\tau_{m}(V)}, \\
\frac{d n}{d t}=\frac{n_{\infty}(V)-n}{\tau_{n}(V)}, \\
\frac{d h}{d t}=\frac{h_{\infty}(V)-h}{\tau_{h}(V)},
\end{gathered}
$$



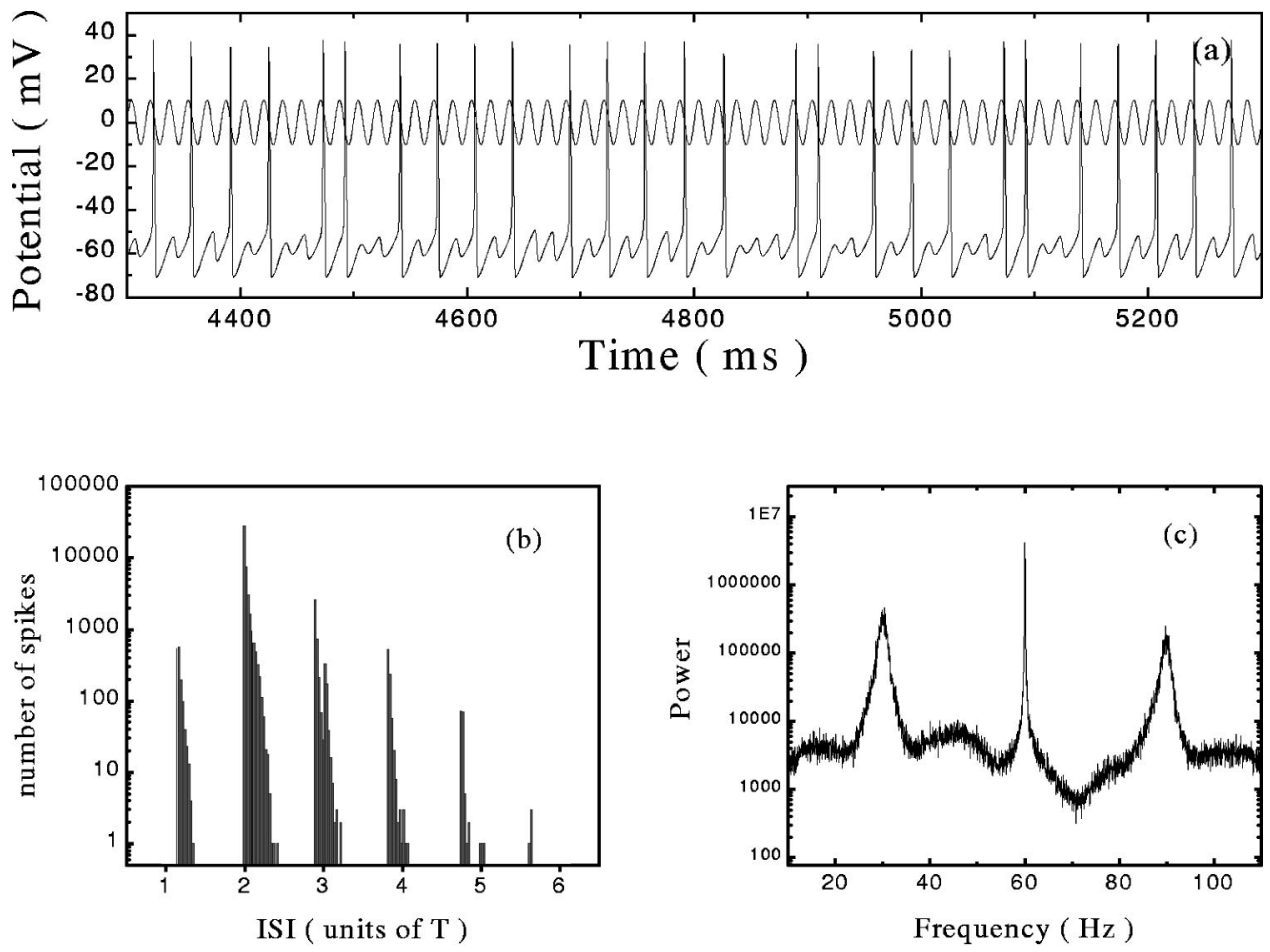

FIG. 1. (a) A time series of membrane potentials of a $\mathrm{HH}$ neuron subjected to a constant bias $I_{0}=3.12$, and a signal with $I_{1}$ $=1.02, f=60 \mathrm{~Hz}$. The amplitude for the signal is enlarged ten times for better viewing. (b) The corresponding interspike interval histogram. (c) The corresponding power spectrum density graph. with

$$
\begin{aligned}
f(V, m, n, h)= & -g_{\mathrm{Na}} m^{3} h\left(V-V_{\mathrm{Na}}\right)-g_{\mathrm{K}} n^{4}\left(V-V_{\mathrm{K}}\right) \\
& -g_{L}\left(V-V_{L}\right),
\end{aligned}
$$

where $V$ is the membrane potential, $m$ and $h$ the activation and inactivation variables of the sodium current, and $n$ the activation variable of the potassium current. The parameters $g_{\mathrm{Na}}, g_{\mathrm{K}}$, and $g_{L}$ are the maximum conductances per surface unit for the sodium, potassium, and leak currents, and $V_{\mathrm{Na}}$, $V_{\mathrm{K}}$, and $V_{L}$ are the corresponding reversal potentials. $m_{\infty}, h_{\infty}, n_{\infty}$ and $\tau_{m}, \tau_{n}, \tau_{h}$ represent the saturation values and the relaxation times of the gating variables. Detailed values of the parameters can be found in Ref. [16]. The system is subject to two external currents $I_{0}$ and $I_{1} \sin (2 \pi f t)$. Because $I_{0}$ is independent of time, changing it is equivalent to changing the reversal potential of leakage channel $V_{L}$ in Eq. (1). In the following simulation, we tune intrinsic oscillations by changing $I_{0} \cdot I_{1} \sin (2 \pi f t)$ is a weak periodic signal with $I_{1}$ and $f$ being the amplitude and frequency of the signal, respectively.

Let us see first how the subthreshold intrinsic oscillation enhances signal transduction (Fig. 1). We choose $I_{1}=1.02$ and $f=60 \mathrm{~Hz}$. This signal is too weak to excite a neuron(the threshold is $\left.I_{1}=1.5\right)$. If a constant bias is applied to the system, the additive constant bias is still unable to excite the neuron if $I_{0}$ is small. In this case, the neuron experiences a subthreshold oscillation. As the constant bias increases, the subthreshold oscillation becomes stronger and stronger. When the bias exceeds the threshold $\left(I_{0}=2.75\right)$, the neuron is excited to output the spike train [Fig. 1(a)]. The neuron does not fire in each period cycle of the external signal, with several cycles being skipped. Importantly, such skipping is irregular and the spike train is stochastic. We plot the ISIH of the spike train in Fig. 1(b). The peaks of the ISIH are roughly multiples of the forcing period, and the envelope of the ISIH decays exponentially except for the first few peaks. Such stochastic coherent characteristics are also seen in the power spectrum density (PSD) of the spike train [Fig. 1(c)]. A main peak located at the forcing frequency and several other peaks located at multiples of the forcing frequency indicate that the input signal is included in the output spike trains. A nonzero SNR, which is calculated from the ratio of the height of the main peak to its nearby background in the PSD, is seen clearly even in the absence of external noise. Due to the deterministic features of the chaotic firing, there are several small peaks at $f^{\prime}=f / n$ (with $n=2,3,4$ ) which represent the spikes with intervals of $T^{\prime}=n T$ ( $T$ is the period of the external forcing). These small peaks are reduced if a weak external noise is applied. Both the ISIH and PSD obtained here are almost the same as those of SR in noisy bistable model, although the dynamics is deterministic here.

We plot the firing rate as a function of $I_{0}$ and $f$ for a weak periodic input signal $\left(I_{1}=1.02\right)$ in Fig. 2, which shows the dynamic behavior near the threshold. When the frequency of the signal ranges from 45 to $65 \mathrm{~Hz}$, the system first experiences chaotic firing as $I_{0}$ increases to cross the threshold. It is in this frequency region that ISR is present. This feature is quite different from the usual SR, due to the fact that the $\mathrm{HH}$ model has specific frequency of intrinsic oscillation which plays a crucial role in ISR.

The dynamical features of the ISR can be seen in Fig. 3, where we plot the maximum Lyapunov exponents $\lambda$, the projection of the membrane potential, and the interspike intervals. Beyond the threshold, regions with positive (chaotic regions) and negative (mode-locked regions) maximum Lyapunov exponents appear alternately as $I_{0}$ increases [Fig. $3(\mathrm{a})$ ], until a steady state is reached [17]. In the chaotic re- 


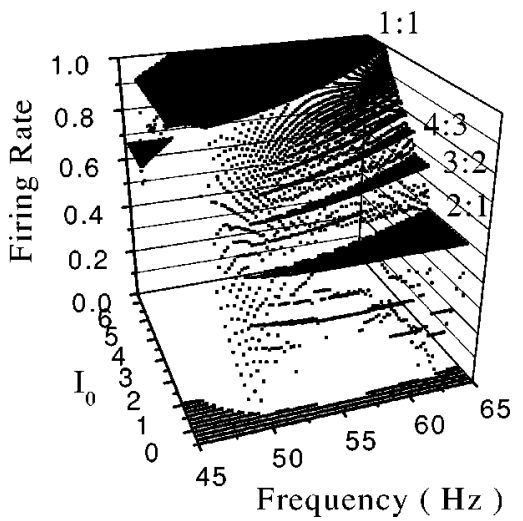

FIG. 2. The mean firing rate for the parameter region of $I_{0}$ vs $f$, with $I_{1}=1.5$. The mean firing rate is calculated by dividing the number of output spikes in 1500 signal periods by 1500 . The labeling $m: n$ on some flat surfaces shows where there are on average $n$ spikes per $m$ signal periods, which is the reciprocal of the firing rate.

gions, the ratio of the number of output spikes to the number of signal periods is irrational. We can also examine this case by the projection of membrane potentials at times separated by the signal period, as plotted in Fig. 3(b). Chaotic regions are those with many projection points and are characterized by multimodal ISI's [Fig. 3(c)]. These results show that chaotic firing behaviors can be obtained by changing the con-

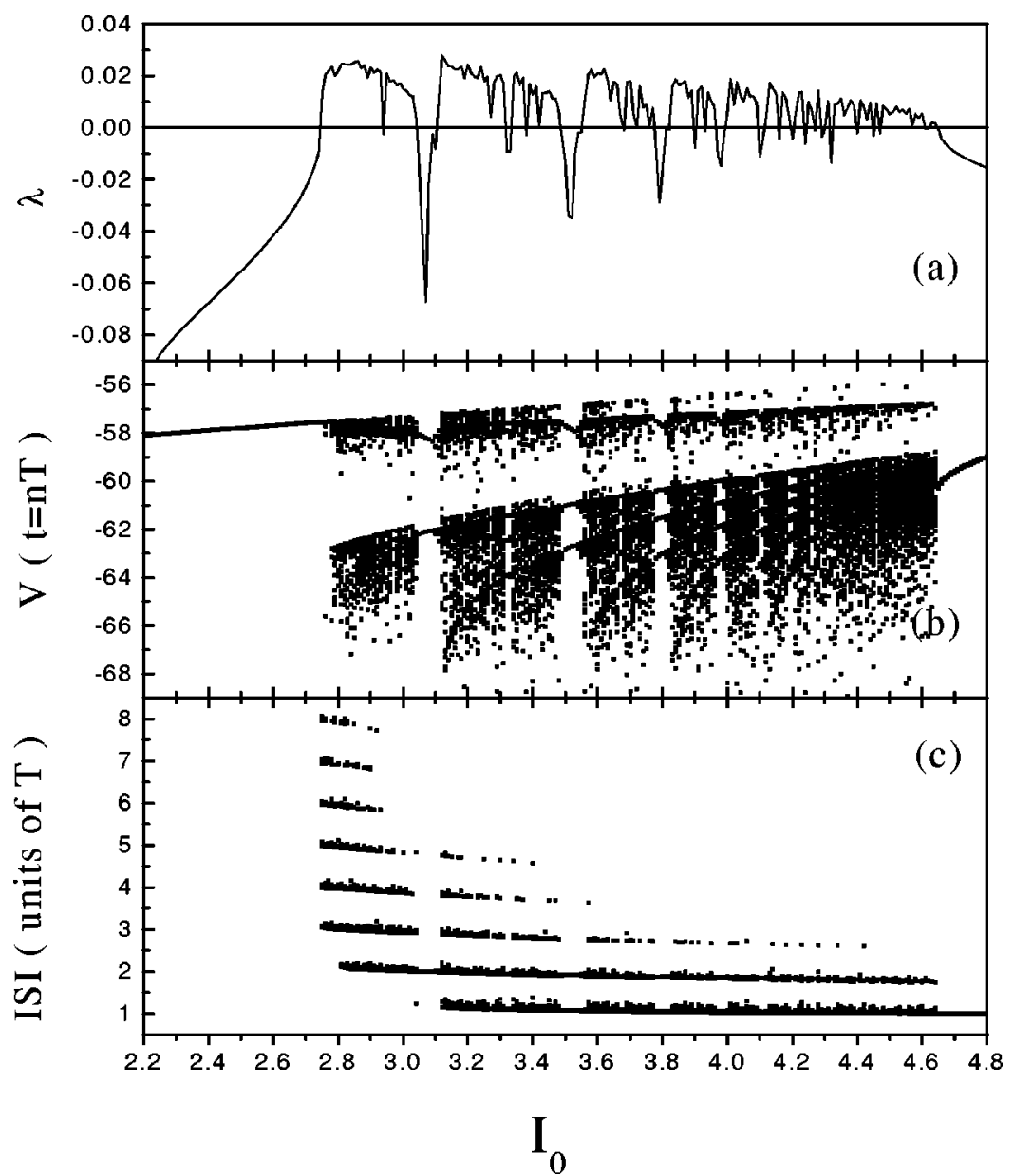

stant bias on a periodically forced $\mathrm{HH}$ neuron.

As shown in Fig. 3, ISR in the $\mathrm{HH}$ model behaves in some manner differently from that in second order nonlinear oscillation models. In the FHN and HR models [4-6], there exists a large region of subthreshold bifurcation where the period doubling bifurcation is obvious; while subthreshold period doubling is hardly observed for the $\mathrm{HH}$ model. In addition, different intrinsic patterns in the $\mathrm{HH}$ model lead to different phase diagrams and dynamical features from those in other models. For example, intrinsic oscillations can be chaotic when tuning $I_{0}$ in the HR model [5], while such chaotic intrinsic oscillations cannot be found in the absence of periodic forcing in the $\mathrm{HH}$ model.

In realistic neural systems, external noises cannot be completed switched off, and thus a mixed effect of external fluctuations and deterministic chaos would be expected. We now consider a system subject to an external noise $\eta(t)$, which satisfies

$$
\frac{d \eta(t)}{d t}=-\frac{\eta(t)}{t_{c}}+\frac{\xi(t)}{t_{c}}
$$

with

$$
\langle\eta(t) \eta(t+\tau)\rangle=\left(D / t_{c}\right) \exp \left(-\tau / t_{c}\right)
$$

FIG. 3. The dynamical behaviors of the neuron as a function of $I_{0}$ with $I_{1}=1.02, f=60 \mathrm{~Hz}$. (a) The maximum Lyapunov exponents $\lambda$, (b) the projections of membrane potentials at times separated by the signal period $T$, and (c) the interspike interval histogram. 


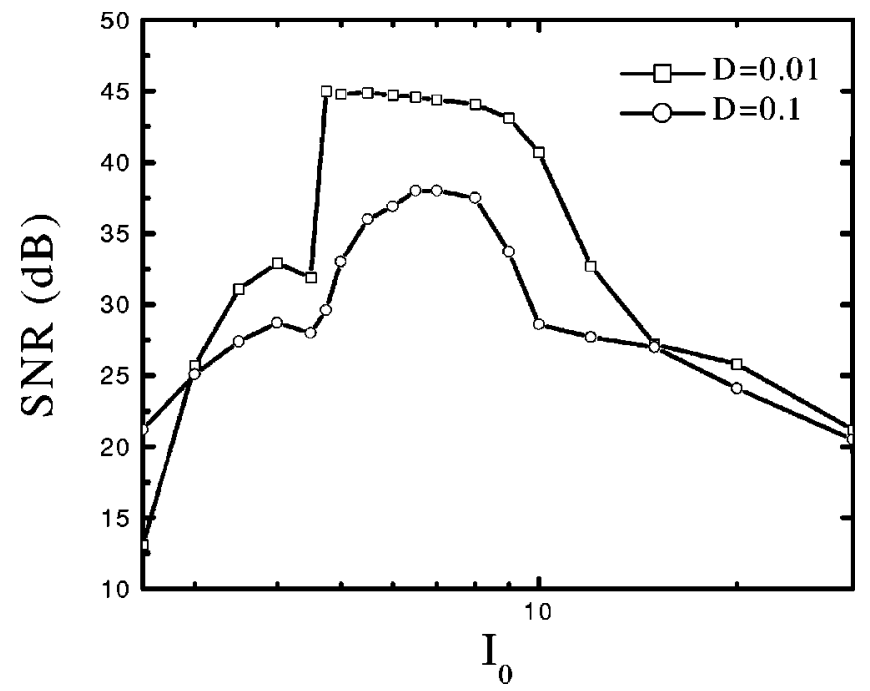

FIG. 4. The output signal-to-noise ratio for different constant bias $I_{0}$ with $I_{1}=1.02, f=60 \mathrm{~Hz}$, with OU noise of $D=0.01$ and 0.1 .

where $\xi(t)$ is the Gaussian white noise with variance $D$ and $t_{c}(=0.1 \mathrm{~ms})$ is the correlation time. Equation (6) describes a zero-mean Ornstein-Uhlenbeck (OU) stochastic process [18].

One feature of SR is to enhance the ability to transmit or encode signals in the system, which can be measured from the signal-to-noise ratio. When there is no noise (not shown here), the peak-to-base ratio of the power spectrum reaches maxima for all mode-locked regions. When a weak OU noise is applied, the output SNR first increases to a maximum and then decreases as $I_{0}$ increases (Fig. 4). Not all combinations of parameters $\left(I_{0}, I_{1}, f\right)$ give this bell-shaped curve, but once the suitable parameters are chosen, the bell-shaped curve can be obtained for any value of noise intensity $D$ provided that $D$ is not too large. If $D$ is very large, the curve becomes flattened. We may split the bell-shaped SNR curve into three regions: the rising region, the top plateau region, and the falling region. In the absence of noise, multimodal firing patterns and mode-locked patterns occur alternately in the rising region; in the presence of noise, the mode-locked states are destroyed so that almost only multimodal states appear. The chaoticity is like an extra noise that decreases the SNR. The falling region is another chaotic firing state: the intrinsic bursting. The output spikes are not related to the signal but to the excitability of the system. The present bellshaped SNR curve may be compared with the effect of SR in various experiments [7-9].

From the SNR, we find that the signal enhancement effect is independent of chaotic noise strength (controlled by deterministic input), and the stochasticity is generated by the intrinsic dynamical property of the excitable system. We can compare this with conventional SR. For conventional SR, similar bell-shaped curves are observed [12]. We note that $I_{0}$ plays a similar role to $D$, although they are obviously different things.

To see and to analyze the role of $I_{0}$ in the parameter range of ISR, we plot the mean firing rate (number of spikes per number of signal periods) versus $I_{0}$ for a moderate noise
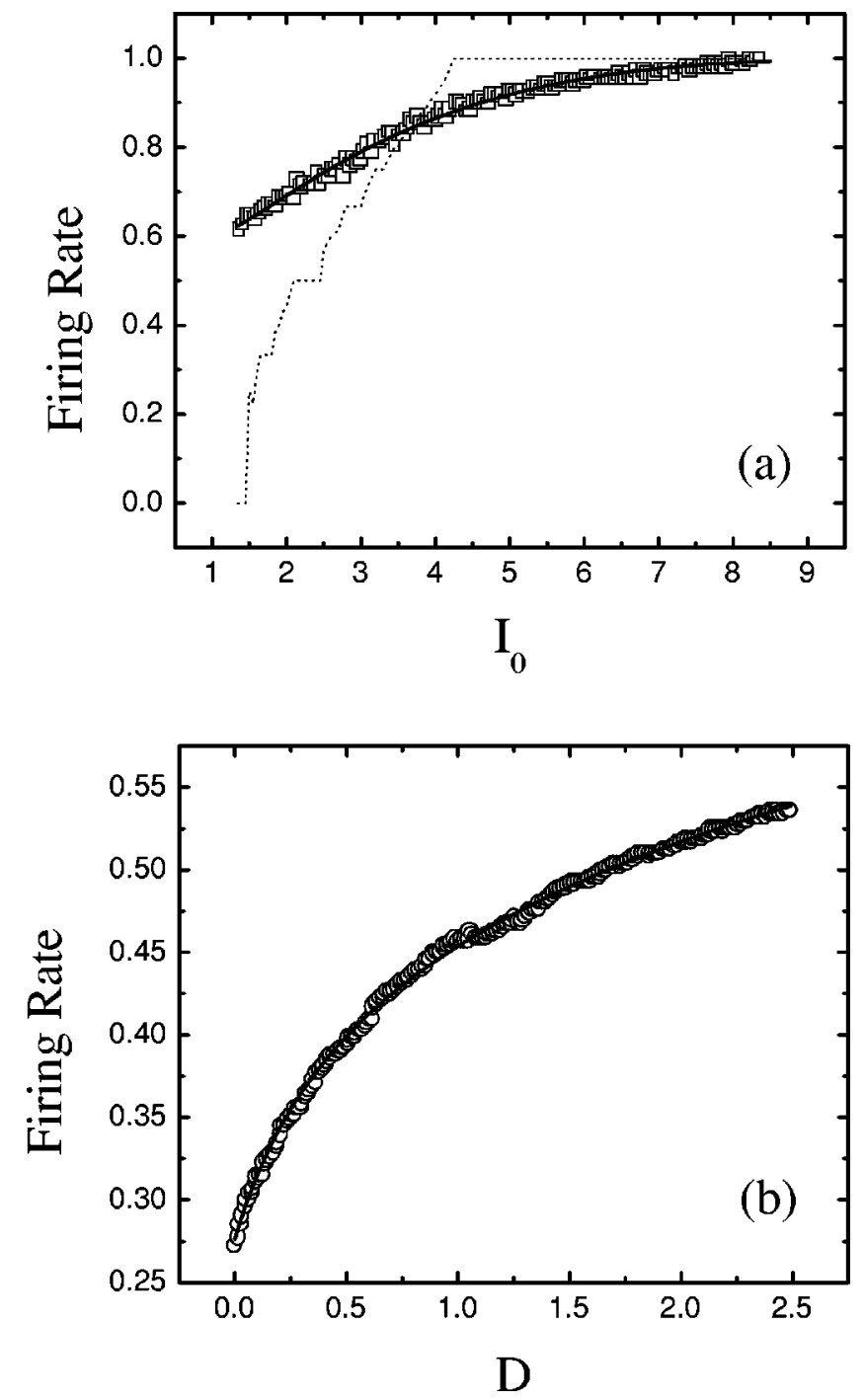

FIG. $5 . I_{1}=1.2$ and $f=60 \mathrm{~Hz}$. The mean firing rate is calculated by the method described in Fig. 2. (a) The mean firing rate vs the constant bias $I_{0}$ with $D=0$ (dotted line) and (scattered squares) and its fitting by Eq. (11) (solid line). (b) The mean firing rate vs the external noise intensity $D$ with $I_{0}=1.58$ (scattered circles), and its fitting by Eq. (10) (solid line).

$D=5$ in Fig. 5(a). In the absence of external noise, there are some mode-locked states where the firing rate is unchanged ("steps") as well as chaotic regions between the flat "steps" [the dotted line in Fig. 5(a)]. The whole structure of the staircase is complicated, but the upward trend is clear. For comparison, we also plot the mean firing rate versus the noise intensity $D$ in Fig. 5(b), where $I_{0}$ is chosen such that the neuron exhibits multimodal firings for $D=0$.

To understand better as well as to compare the data in Figs. 5(a) and 5(b), it is helpful to employ a Kramer-type formula for the probability rate of state switching in multistable systems when the system is periodically forced $[9,19]$ :

$$
R(t)=\exp \left\{\left(-U / D_{t o t}\right)[1-a \cos (2 \pi f t)]\right\},
$$

where $U$ is an effective barrier height, $D_{t o t}$ is the effective total noise intensity, and $a$ is a constant. The mean firing rate can be obtained as 


$$
R=\langle R(t)\rangle=\exp \left[\left(-U / D_{t o t}\right)\right] B_{0}\left(a U / D_{t o t}\right)
$$

where $B_{0}$ is the zeroth order modified Bessel function. From Eq. (9), we are motivated to fit Fig. 5(b) with

$$
R(D)=A\left(D+D_{0}\right)^{E} \exp \left[-B /\left(D+D_{0}\right)\right]
$$

where $A=0.461, B=0.0745, D_{0}=0.270$, and $E=0.180$. We find that $D_{0}$ is nonzero, which implies the existence of background chaos that produces the multimodal firing when $D$ $=0$. For $D>0$, the chaos acts like an extra noise. Since it is expected that the effect of changing $I_{0}$ will be similar to that of changing the noise intensity in some sense, we may try to fit Fig. 5(a) by

$$
R=A_{1}\left(I_{0}\right)^{E_{1}} \exp \left(-B_{1} / I_{0}-I_{z}\right)
$$

where $A_{1}=14.2, B_{1}=11.8, I z=-2.72$, and $E_{1}=-0.751$. The fitting appears to be acceptable and the similarity between changing $I_{0}$ and $D$ can be seen from the fittings Eqs. (10) and (11). In fact, $I_{0}$, together with the sinusoidal signal, drives the system into the chaotic regime. The mixed effect of the total input $I$ and the excitability produces a subthreshold chaos that plays the role of noise and enhances the signal detection through a mechanism like SR, so that an undetectable weak signal may become detectable (at least partially). In addition, comparing Eq. (11) with Eq. (9), one can see that increasing $I_{0}$ has a similar effect as decreasing $U$, and the effect is nonlinear and very complicated.

The noise, signal, and barrier are three elements of SR. In bi- or multistable systems, since the constant bias affects the barrier height linearly, no chaotic background can be produced. Therefore external noise is necessary for SR in these systems. In excitable systems, however, $I_{0}$ changes the effective barrier height in a nonlinear way, which explains why there exists the effect of SR even when the external noise is switched off. Certainly, the excitability is a key element in this effect. In excitable systems like the HH neuronal system, the effective barrier height is not merely a fixed threshold but dependent on the recovery, as the system cannot give full responses during this stage. The recovery rate is not unchanged after each discharge of the membrane potential, rather it is dependent on the phase of the input current when firing happens. For certain parameter values of $I_{0}, I_{1}$, and $f$, the recovery time scales can become irregular and the corresponding oscillation is that of the subthreshold chaos mentioned above. For appropriate parameters, the fluctuation can become chaotic and acts like a noise to enhance the trans- duction of deterministic input via the mechanism of SR. As a result, there exists a stochastic resonance without noise (i.e., ISR) in excitable systems.

In conventional SR, the noise and the signal can be distinguished as two different inputs. However, we cannot separate the subthreshold chaos from the signal in our case. If there is only $I_{0}$ or the signal, we can observe either a constant or a periodically oscillating subthreshold membrane potential. The chaos comes from the mixed effect of $I_{0}$, the signal, and the excitability. $I_{0}$ is merely a parameter used for observing the ISR in our simulation. We can vary $I_{1}$ to observe a similar effect. However, the necessity of the signal implies a kind of self-organization in neuronal systems. The input signal, together with the constant bias, produces a chaos that enhances the detection of the signal itself. Although a random number of cycles are skipped, the timing of the spike trains is still dependent on the signal period, and thus the precise timing of the stimulation is remembered. Recently, it was indicated that some sensory information is encoded in time, rather than firing rate [20], so that multimodal firing patterns can carry information as usefully as phaselocked ones. For a certain weak sinusoidal signal, we may consider $I_{0}$ as the control parameter, which, biologically, could be either a constant part of the internal noise or an intrinsic electrical property of the neuron. In the former case, the internal noise is mainly the stimulus from other neurons in the network, causing a subthreshold fluctuation in the membrane potential [21]. In the latter case, the electrical properties including the constant bias of neurons can be modulated by a stimulus from the environment [22]. This tuning effect is easily found in sensory neurons, and based on it the organism can adapt to its environment.

In summary, we have found that excitability is important in detecting weak signals in the $\mathrm{HH}$ model, via the mechanism of ISR. This interesting phenomenon is absent in the usual bi- or multistable systems. The resonant oscillation between the output and weak external forcing in the absence of external noise is enhanced by subthreshold intrinsic oscillations and is demonstrated in a multimodal ISIH, the SNR, and the statistics of the mean firing rate. Rather than relying only on the external noise, a neural system may tune itself to be chaotic to encode weak signals.

This work was supported by a CRCG grant at the University of Hong Kong. Y.W. acknowledges the support of the Pacific Institute for Mathematical Sciences and an NSERC grant.
[1] V. Anishchenko, F. Moss, A. Neiman, and L. SchimanskyGeier, Phys. Usp. 42, 7 (1999); L. Gammaitoni, P. Hänggi, P. Jung, and F. Marchesoni, Rev. Mod. Phys. 70, 223 (1998); A. R. Bulsara and L. Gammaitoni, Phys. Today 49 (3), 39 (1996); D. Wiesenfield and F. Moss, Nature (London) 373, 33 (1995).

[2] R. Siegel, Physica D 42, 385 (1990); N. Y. S. Kiang, Handbook of Physiology, Vol. III (American Physiology Society, Bethesda, MD, 1984), Pt. 2, Chap. 5, p. 639; W. H. Talbot, I.
Darian-Smith, H. H. Kornhuber, and V. B. Mountcastle, J. Physiol. (London) 31, 301 (1968); T. Ogawa, P. O. Bishop, and W. R. Levik, J. Neurophysiol. 6, 2 (1966).

[3] A. Longtin, A. R. Bulsara, and F. Moss, Phys. Rev. Lett. 67, 656 (1991); A. Longtin, A. R. Bulsara, D. Pierson, and F. Moss, Biol. Cybern. 70, 569 (1994).

[4] D. T. Kaplan, J. R. Clay, T. Manning, L. Galss, M. R. Guevara, and A. Shrier, Phys. Rev. Lett. 76, 4074 (1996). 
[5] W. Wang, Y. Q. Wang, and Z. D. Wang, Phys. Rev. E 57, R2527 (1998); Y. Q. Wang, Z. D. Wang, and W. Wang, J. Phys. Soc. Jpn. 69, 276 (2000).

[6] Y. Q. Wang, Z. D. Wang, and W. Wang, J. Phys. Soc. Jpn. 67, 3601 (1998).

[7] J. E. Levin and J. P. Miller, Nature (London) 380, 165 (1996); B. J. Gluckman, T. I. Netoff, E. J. Neel, W. L. Ditto, M. L. Spano, and S. J. Schiff, Phys. Rev. Lett. 77, 4098 (1996); D. Petracchid, M. Pellegrini, M. Pellegrino, M. Barbi, and F. Moss, Biophys. J. 66, 1844 (1994).

[8] J. K. Douglass, L. Wilkens, E. Pantazelou, and F. Moss, Nature (London) 365, 337 (1993); E. Pantazelou, C. Dames, F. Moss, J. K. Douglass, and L. Wilkens, Int. J. Bifurcation Chaos Appl. Sci. Eng. 5, 101 (1995); J. K. Douglas and F. Moss, Nav. Res. Rev. XLV, 30 (1993).

[9] K. Wiesenfeld, D. Pierson, E. Pantazelou, C. Dames, and F. Moss, Phys. Rev. Lett. 72, 2125 (1994).

[10] V. S. Anishchenko, A. B. Neiman, and M. A. Safanova, J. Stat. Phys. 70, 183 (1993).

[11] A. Longtin, Phys. Rev. E 55, 868 (1997); A. Neiman, P. Saparin, and L. Stone, ibid. 56, 270 (1997).

[12] J. J. Collins, C. C. Chow, A. C. Capela, and T. T. Imhoff, Phys. Rev. E 54, 5575 (1996).

[13] Y. Q. Wang, D. T. W. Chik, and Z. D. Wang, Phys. Rev. E 61, 740 (2000); F. Liu, J. F. Wang, and W. Wang, ibid. 59, 3453 (1999); W. Wang and Z. D. Wang, ibid. 55, 7379 (1997).

[14] U. Feudal, A. Neiman, X. Pei, W. Wojtenck, H. A. Braun, M. T. Huber, and F. Moss, Chaos 10, 231 (2000); H. A. Braun, M. T. Humber, M. Dewald, K. Schäfer, and K. Voigt, Int. J. Bifurcation Chaos Appl. Sci. Eng. 8, 881 (1998); Note that two slow ionic currents exist in the modified $\mathrm{HH}$ model used in these papers, which are absent in the usual $\mathrm{HH}$ model. The two additional time scales in their model lead to many different patterns in the spontanuous oscillations. For example, a multimodal ISIH can be observed even in the absence of external periodic forcing. In addition, the parameters of their model seem to be set without threshold.

[15] J. R. Cooper, F. E. Bloom, and R. H. Roth, The Biochemical Basis of Neuropharmacology, 6th ed. (Oxford University Press, New York, 1991); M. E. Hasselmo, Behav. Brain Res. 67, 1 (1995); J. M. Fellous and C. Linster, Neural Comput. 10, 771 (1998).

[16] A. L. Hodgkin and A. F. Huxley, J. Physiol. (London) 117, 500 (1952); D. Hansel, G. Mato, and C. Meunier, Phys. Rev. E 48, 3470 (1993).

[17] This 1:1 state does not necessarily exist for other combinations of parameters $I_{0}, I_{1}, f$.

[18] P. V. E. McClintock and F. Moss, in Noise in Nonlinear Dynamical Systems, edited by F. Moss and P. V. E. McClintock (Cambridge University Press, Cambridge, England, 1989), Vol. 3, p. 243.

[19] W. M. Siebert, Proc. IEEE 58, 723 (1970).

[20] W. R. Softky, Curr. Opin. Neurobiol. 5, 239 (1995); P. König, A. K. Engel, and W. Singer, Trends Neurosci. 19, 130 (1996); Y. Q. Wang and Z. D. Wang, Phys. Rev. E 62, 1063 (2000).

[21] Many experiments have reported that such fluctuation contains a nonrandom component [see, for example, P. Faure and $\mathrm{H}$. Korn, Proc. Natl. Acad. Sci. U.S.A. 94, 6506 (1997); D. Desmaisons, J. D. Vincent, and P. M. Lledo, J. Neurosci. 19, 10727 (1999); R. Amir, M. Michaelis, and M. Devor, ibid. 19, 8589 (1999)]. So we might expect that there should be a constant component, which is about the average of the fluctuation.

[22] The Neuron: Cell and Molecular Biology, 2nd ed., edited by I. B. Levitan and L. K. Kaczmarek (Oxford University Press, New York, 1997), Chap. 12. 